\title{
Load Profile Analysis of Medium Voltage Regulating Transformers on Battery Energy Storage Systems (BESS)
}

\author{
Robert Beckmann \\ Energy Systems Technology \\ German Aerospace Center \\ Institute of Networked Energy Systems \\ Oldenburg, Germany \\ robert.beckmann@dlr.de \\ Frank Schuldt \\ Energy Systems Technology \\ German Aerospace Center \\ Institute of Networked Energy Systems \\ Oldenburg, Germany \\ frank.schuldt@dlr.de
}

\author{
Ewald Röben \\ swb Erzeugung $A G$ \& Co. $K G$ \\ Bremen, Germany \\ ewald.roeben@swb-gruppe.de
}

\author{
Jens Clemens \\ swb Services $A G \&$ \&.$K G$ \\ Bremen, Germany \\ jens.clemens@swb-gruppe.de
}

\begin{abstract}
Battery Energy Storage Systems (BESS) already cover a large part of the Frequency Containment Reserve (FCR) in Germany. If these are built at locations of conventional power plants, the infrastructure available there may be utilized. In this paper we investigate how the secondary voltages of two medium voltage regulating transformers differ by means of a model-based analysis. The first transformer is the auxiliary power transformer of a coal-fired power plant. The second, identical transformer connects the hybrid power plant $\mathrm{HyReK}$ to the medium voltage grid to provide FCR. As an outcome of the model-based investigations we give an estimate of the voltages to be expected during one year and whether they require frequent changes of the tap changer position. We first implement the underlying network connection model in OpenModelica and then export the physical equation set to the Computer Algebra System Maple ${ }^{\mathrm{TM}}$. We also investigate the effect of different system parameters on the secondary side voltage and discuss the results.
\end{abstract}

Index Terms-Battery Energy Storage Systems, Power-to-Heat, frequency containment reserve, electric load profile, sensitivity analysis, HyReK

\section{INTRODUCTION}

Unforeseeable changes in the generation and consumption of electrical energy lead to a change in the grid frequency. There are various control mechanisms that compensate for these deviations. One is the Frequency Containment Reserve (FCR). It compensates deviations of the grid frequency from $50 \mathrm{~Hz}$ very quickly, but only to a limited extent [1].

The $605 \mathrm{MW}$ FCR power tendered in Germany is already largely provided by Battery Energy Storage Systems (BESS). By the end of 2018, BESS with a cumulative power of $402 \mathrm{MW}$ had been installed. The authors in [2] expect that

This work has been funded by the German Federal Ministry for Economic Affairs and Energy (BMWi) under the grant no. 03ET6147C and is part of the project "HyReK 2.0 - Hybrid Regulating Power Station".

\author{
Karsten von Maydell \\ Energy Systems Technology \\ German Aerospace Center \\ Institute of Networked Energy Systems \\ Oldenburg, Germany \\ karsten.maydell@dlr.de
}

after completion of the prequalification procedures up to $63 \%$ of the FCR will be provided by BESS.

In 2018, swb Erzeugung $A G \& C o . K G$ constructed and commissioned the hybrid control power plant (Hybridregelkraftwerk) HyReK in Bremen Hastedt for the provision of $18 \mathrm{MW}$ FCR power. The HyReK combines a $14.244 \mathrm{MWh}$ BESS with a power-to-heat option. This hybrid approach allows negative control power to be fed from the power grid into the district heating grid in the event of over-frequency. By this sector coupling the required battery size could be reduced and in addition the surplus energy in the power grid is used sensibly, thus resulting in a reduction of $\mathrm{CO}_{2}$ emissions.

If BESS are installed at existing power plant sites, components of the existing infrastructure may be used or reused. However, the question arises how the current and future usage patterns differ.

\section{Problem Formulation}

This paper compares the use of two medium voltage regulating transformers (MVT). The first one $\left(\mathrm{MVT}_{\text {aux }}\right)$ is used to supply a power plant with auxiliary power. Via the second, identical transformer $\left(\mathrm{MVT}_{\mathrm{FCR}}\right)$, the FCR power is fed into the power grid or drawn from it. The load profile for $\mathrm{MVT}_{\text {aux }}$ is available from 19 September 2018 to 18 September 2019. For $\mathrm{MVT}_{\mathrm{FCR}}$ no comparable load profile is available yet. For a comparison, the theoretical FCR power load curve is therefore calculated from the regulations in [3] and [1] and the grid frequency during this period. When calculating the primary control power from the grid frequency, we only use the frequency dead band of the degrees of freedom specified in [3].

If the power is positive, it is drawn from the grid, if the power is negative, it is fed into the grid accordingly. We will use these signs throughout this article. In the case of 


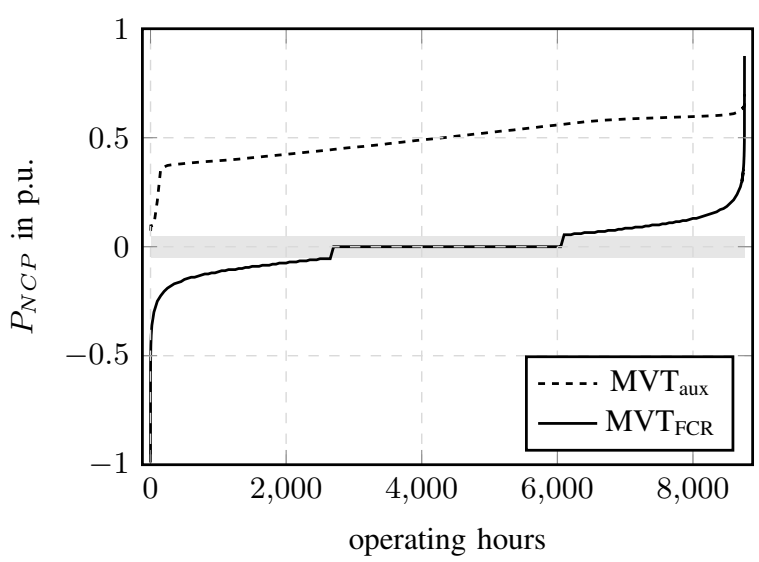

Fig. 1. Ordered annual load profiles for auxiliary power transformer (solid line) and FCR transformer (dotted line) for 365 days - The light grey zone indicates the FCR dead band where a power of 0 p.u. is assumed.

the HyReK this means that if the power is positive, the batteries are charged or the power is transferred to the powerto-heat option and if the power is negative, the batteries are discharged.

Both load profiles are shown in Fig. 1 as ordered annual load profiles. The auxiliary power transformer $\mathrm{MVT}_{\mathrm{aux}}$ has always a positive load, which is at no time less than 0.07 p.u. The FCR transformer $\mathrm{MVT}_{\mathrm{FCR}}$ would conduct power between -1 p.u. and 0.86 p.u. However, $39 \%$ of the time the power is zero since the frequency is in the dead band. If the direction of energy flow is neglected, i.e. the absolute values of the power are considered, it can be seen that the power of $\mathrm{MVT}_{\text {aux }}$ is less than 0.6 p.u. in $90 \%$ of the operating hours. With $\mathrm{MVT}_{\mathrm{FCR}}$ the power is even less than 0.15 p.u. in $90 \%$ of the operating hours. Integrating the energy flows, $\mathrm{MVT}_{\mathrm{aux}}$ conducts about seven times more energy. The real load curve of $\mathrm{MVT}_{\mathrm{FCR}}$ will differ slightly from this, due to the fact that the FCR operating strategy can use the degrees of freedom mentioned in [3].

After we have shown that the load profiles of both types of use differ significantly, we are now investigating how these differences affect the secondary side voltage of the medium voltage transformer and whether increased switching of the tap changer in FCR operation is to be expected.

The required power is set at the grid connection point by varying the current. As an undesired side effect, the voltage also changes slightly. The extent of this voltage change depends on the current and the structure and state of the network under consideration. In addition to regulating transformers, various other measures are used to compensate for undesirable voltage deviations. In transmission networks, for example, compensators are used [4], [5] and in distribution networks the connected generators must have features for static voltage regulation [6], [7].

The HyReK is connected to the $110 \mathrm{kV}$ grid via two transformers. The medium voltage transformer translates the HyReK nominal voltage from $6.25 \mathrm{kV}$ to $10 \mathrm{kV}$. The high volatge transformer then translates from $10 \mathrm{kV}$ to $110 \mathrm{kV}$. Both transformers are designed as regulating transformers.

This article focuses on regulating transformers with variable transmission ratio where the secondary side voltage is

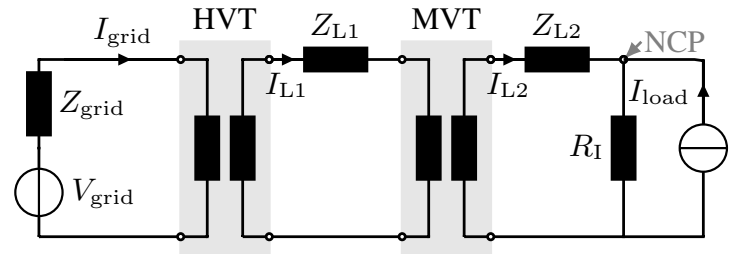

Fig. 2. Single-phase equivalent circuit diagram of the grid connection

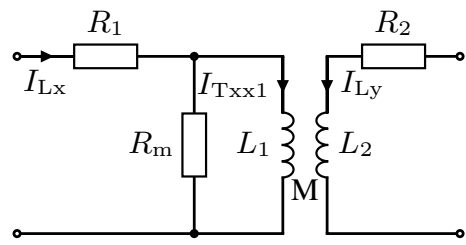

Fig. 3. Single phase $\mathrm{T}$ equivalent circuit diagram of a lossy transformer

measured. If this voltage deviates from the nominal value by more than an admissible amount, the tap changer position is increased or decreased under load [8], [9]. These tap changers are also referred to as On Load Tap Changer (OLTC). The transformers $\mathrm{MVT}_{\mathrm{aux}}$ and $\mathrm{MVT}_{\mathrm{FCR}}$ each have a OLTC with 19 steps. Adjacent positions differ in their primary side nominal voltage by $0.2 \mathrm{kV}$.

To answer the question how the usages in auxiliary power operation and in FCR operation effect the secondary side voltage, we assume both transformers to be connected to the high voltage grid via the same topology. Consequently, one model is sufficient, in which the profile of the feed or load current is adapted.

\section{MODEL DESCRIPTION}

This section describes the model for the grid connection (Fig. 2) consisting of the load (current source), the cable to the medium voltage transformer (impedance), the medium voltage transformer, the cable to the high voltage transformer (impedance), the high voltage transformer (HVT) and the grid. This grid connection model is valid for the analysis of the usage of the MVT as an auxiliary power transformer and as a transformer for FCR operation.

\section{A. Model Structure}

We only investigate scenarios with symmetrical loads, therefore the single-phase equivalent circuit diagram in Fig. 2 is sufficient. In the model the current $I_{\text {load }}$ is to be interpreted as input. The load, for example the BESS, sets it in such a way that with the voltage measured at the NCP, the desired power is transmitted. The grid connection model is described by a differential equation system with the five mesh currents $I_{\text {grid }}$, $I_{H V T}, I_{L 1}, I_{M V T}, I_{L 2}$ as states. We omit the derivation and the imprint of the model equations and instead want to briefly discuss the equivalent circuit diagram of the transformer.

The reduction of the grid connection model to one phase enables the use of the $\mathrm{T}$ equivalent circuit diagram of lossy transformers (Fig. 3). This widely used equivalent circuit diagram is also given in [9], for example. Usually transformers are characterized by parameters such as iron and copper losses 
TABLE I

IMPEDANCES IN THE GRID CONNECTION MODEL

\begin{tabular}{|c|c|c|c|c|c|}
\hline & $\mathbf{Z}_{\text {grid }}$ & $\mathbf{Z}_{\mathbf{L 1}}$ & $\mathbf{Z}_{\mathbf{L 2}}$ & $\mathbf{R}_{\mathbf{I}}$ & unit \\
\hline resistance & 0.301 & 0.015 & 0.005 & $10 \mathrm{e} 6$ & $\Omega$ \\
inductance & 9.581 & 0.064 & 0.022 & & $\mathrm{mH}$ \\
\hline
\end{tabular}

and short-circuit voltage. These descriptive parameters need to be transformed into the parameters of the elements of the equivalent circuit diagram in Fig. 3. For this purpose, we have used the relationships also given in [10]. The used equations are described in the remainder of this subsection.

The resistors $R_{1}, R_{2}$ and $R_{m}$ can be calculated directly from the copper and iron losses $P_{C u}$ and $P_{F e}$ and the nominal apparent power $S_{\text {nom }}$ and the primary and secondary voltages $V_{\text {pri }}$ and $V_{\text {sec }}$.

$$
\begin{aligned}
R_{1} & =\frac{P_{C u}}{2} \frac{V_{p r i}^{2}}{S_{n o m}^{2}} \\
R_{2} & =\frac{P_{C u}}{2} \frac{V_{\text {sec }}^{2}}{S_{n o m}^{2}} \\
R_{m} & =\frac{V_{p r i}^{2}}{P_{F e}}
\end{aligned}
$$

It is assumed that the power losses are distributed equally over the primary and secondary voltage side of the transformer. To calculate the inductances $L_{1}$ and $L_{2}$ and the mutual inductance $M$, the rated magnetizing reactance $X_{m}$ and the rated shortcircuit reactance $X_{S C}$ are required:

$$
\begin{aligned}
X_{m} & =\left(\sqrt{\left(\frac{I_{0}}{100}\right)^{2}-\left(\frac{P_{F e}}{S_{n o m}}\right)^{2}}\right)^{-1} \\
X_{S C} & =\sqrt{\left(\frac{V_{S C}}{100}\right)^{2}-\left(\frac{P_{C u}}{S_{n o m}}\right)^{2}} .
\end{aligned}
$$

The inductances and the mutual inductance are set to

$$
\begin{aligned}
L_{1} & =\frac{X_{m}+\frac{1}{2} X_{S C}}{\omega S_{n o m}} V_{p r i}^{2} \\
L_{2} & =\frac{X_{m}+\frac{1}{2} X_{S C}}{\omega S_{n o m}} V_{s e c}^{2} \\
M & =\frac{X_{m}}{\omega S_{n o m}} V_{\text {pri }} V_{\text {sec }} .
\end{aligned}
$$

\section{B. Parameterization}

The resistances and inductances of the cables are calculated from the estimated cable lengths and the primary line constants from [11]. Their values are given in Table I.

We calculated the parameters of the MVT with only a few assumptions directly from the data sheet. We only know the nominal apparent power of the high voltage transformer, the remaining parameters are set according to the specifications in [4] and [11]. In Table II the parameters of the transformer models are given. For each transmission ratio (tap position) a parameter set is calculated using the equations (1)-(8), using the values and units given in Table II.

The grid voltage is $V_{\text {grid }}=110 \mathrm{kV}$ (RMS, phase to phase), the secondary side rated voltage of the medium voltage transformer is $V_{M V \text {,sec }}=6.25 \mathrm{kV}$ (RMS, phase to phase).
TABLE II

PARAMETERS OF THE HIGH VOLTAGE AND MEDIUM VOLTAGE TRANSFORMERS

\begin{tabular}{|c|c|c|c|c|}
\hline & symbol & HVT & MVT & unit \\
\hline nom. apparent power & $S_{n o m}$ & 35 & 18 & MVA \\
pri. side nom. voltage & $V_{\text {pri }}$ & 110 & $11.8 / 0.2 / 8.2$ & $\mathrm{kV}$ \\
sec. side nom. voltage & $V_{\text {sec }}$ & 10 & 7 & $\mathrm{kV}$ \\
short circuit voltage & $V_{S C}$ & 12.3 & 8.25 & $\%$ \\
idling current & $I_{0}$ & 0.14 & 0.14 & $\%$ \\
iron losses & $P_{F e}$ & 0.014 & 0.016 & $\mathrm{MW}$ \\
copper losses & $P_{C u}$ & 0.135 & 0.100 & $\mathrm{MW}$ \\
\hline
\end{tabular}

\section{Implementation}

We have modeled the grid connection topology (Fig. 2) in OpenModelica [12] and in Simscape Electrical [13] with the Specialized Power Systems components. In selected test scenarios we have verified that both implementations produce the same results. From the implementation in OpenModelica we exported the differential-algebraic equation system and symbolically combined it via the computer algebra system Maple $^{\mathrm{TM}}$ [14] to a system of ordinary differential equations of fifth order. For the analysis in the stationary operating points we finally converted the system for $\omega=2 \pi f$ into the complex phasor representation. From its solutions (currents) the remaining model quantities are calculated. Among other things, this allowed us to examine the effect of the tap changer position, line resistance and reactive power on the voltage at the grid connection point. Simulations of the Simscape Electrical implementation confirmed the results in Maple ${ }^{\mathrm{TM}}$.

This procedure is not mandatory. The results we show in the following section can also be obtained by performing simulation studies in OpenModelica or Simulink ${ }^{\mathrm{TM}}$ where the parameters in question are varied. With this approach, however, the pre- and post-processing is much more cumbersome.

\section{ANALYSIS}

The power is set via the load current $I_{\text {load }}$. Depending on this current, a voltage $V_{M V, s e c}$ is obtained at the secondary side of the MVT.

\section{A. Tap Postion}

Figure 4 shows the voltage $V_{M V \text {,sec }}$ for selected positions of the tap changer over the active power

$$
P_{N C P}=\operatorname{Re}\left(\frac{1}{2} \cdot V_{\mathrm{NCP}} \cdot \bar{I}_{\mathrm{L} 2}\right)
$$

at the grid connection point.

In position 4 the transmission ratio of the medium voltage transformer is $r_{\mathrm{MV}}=\frac{11.2 \mathrm{kV}}{7 \mathrm{kV}}=1.6$.

The voltage $V_{M V \text {,sec }}$ varies between 1.002 p.u. and 0.97 p.u., it has its maximum at $P_{N C P}=-0.38$ p.u.. Switching the tap changer position up or down results in a secondary side voltage increase or decrease of 0.018 p.u. which is practically constant over the entire power range.

In tap changer position 5 the voltage $V_{M V \text {,sec }}$ can vary between 1.02 p.u. and 0.99 p.u.. In this position, the voltage deviation is therefore less than $2 \%$ over the entire power range. 


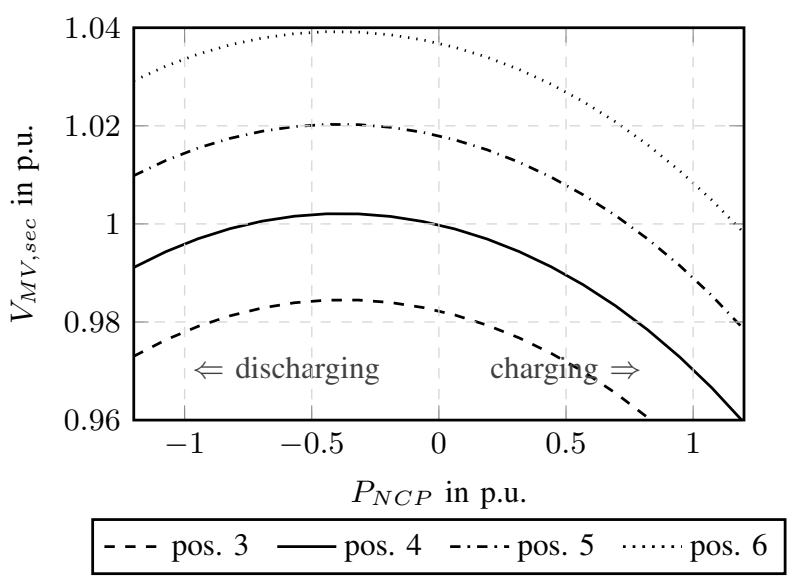

Fig. 4. Voltage at secondary side of the medium voltage transformer $T_{M V}$ for four different tap positions

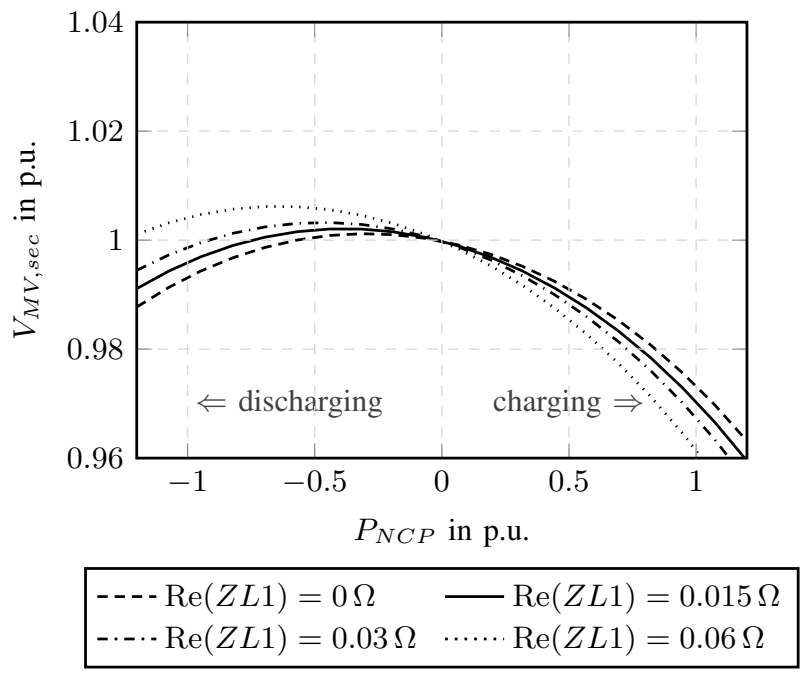

Fig. 5. voltage at secondary side of the medium voltage transformer for four different line resistances

\section{B. Cable Resistance}

Based on the estimated cable length and the specifications in [11] we have assumed a resistance of $R_{Z L 1}=0.015 \Omega$ for the ohmic part of the impedance between the transformers (see Table I). To determine the influence of the parameter on the secondary voltage of the medium voltage transformer, we have varied the resistance between $0 \Omega$ and $0.06 \Omega$. Figure 5 shows that the cable resistance has little influence on the voltage $V_{M V \text {,sec. }}$ The maximum deviation between the voltages at $0 \Omega$ and $0.06 \Omega$ is less than 0.012 p.u. in the entire power range.

\section{Reactive Power}

Besides the active power, the reactive power also influences the voltage $V_{M V \text {,sec }}$. Fig. 6 shows the voltage over the active power for different reactive powers. Already a reactive power of $Q_{N C P}=-0.1$ p.u. at $P_{N C P}=0$ p.u. raises the voltage by 0.017 p.u.. Further analysis shows that in the network topology under consideration, the sensitivity of the voltage $V_{M V \text {,sec }}$ with respect to reactive power is about 15 times greater than with respect to active power. This property might be used to

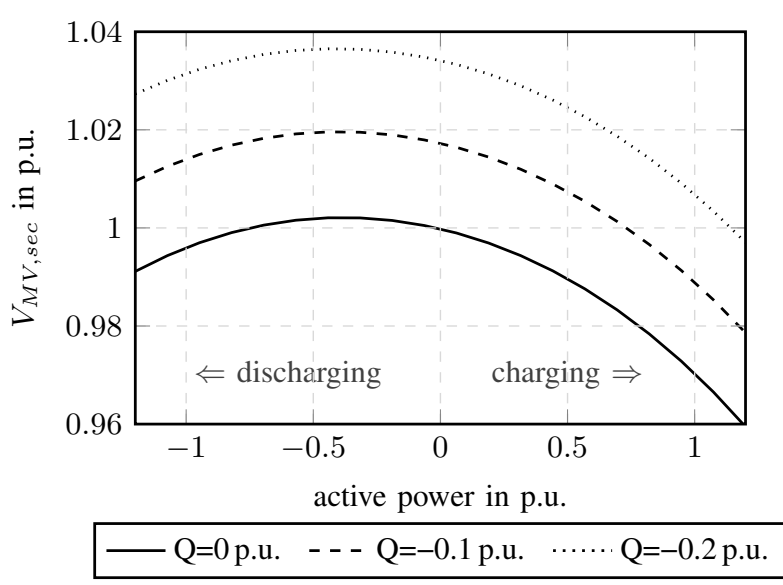

Fig. 6. Voltage at secondary side of the medium voltage transformer MVT for three different constant reactive powers

TABLE III

MINIMA, MAXIMA, MEAN VALUES OF THE VOLTAGES $V_{M V \text {, sec }}^{a u x}$ AND $V_{M V, s e c}^{F C R}$ IN P.U.

\begin{tabular}{|c|c|c|c|}
\hline & min & max & mean \\
\hline$V_{M V, s e c}^{a u x}$ & 0.983 & 0.999 & 0.9896 \\
$V_{M V, \text { sec }}^{F C R}$ & 0.976 & 1.002 & 0.9996 \\
\hline
\end{tabular}

adjust the reactive power so that the voltage is kept within an acceptable band.

\section{Ordered Annual Voltage Profile}

From the measured load of the auxiliary power transformer $\mathrm{MVT}_{\mathrm{aux}}$ and the expected load of the FCR transformer $\mathrm{MVT}_{\mathrm{FCR}}$ we calculate the secondary side voltages $V_{M V \text {,sec }}$, which occur in the recording period. For the calculation we use the relationship between power $P_{N C P}$ and the secondary voltages $V_{M V, \text { sec }}$ shown in Fig. 4, for tap changer position 4.

The minimum, maximum and mean values of the calculated voltages are listed in Table III, the voltages are shown as ordered annual voltage profiles in Fig. 7.

On the left, the graph of $V_{M V \text {,sec }}^{F C R}$ asymptotically approaches the ordinate axis. Thus it is not visible in this figure that the voltage deviates more than $2 \%$ from 1 p.u. in about 100 seconds of these 8760 hours only. This deviation is due to a frequency event on 24 January 2019 at 5 a.m. (CET).

\section{Discussion}

The low losses of the cables and the transformers produce only small voltage deviations at the grid connection point and the secondary side of the MVT. Furthermore, the analyses in the previous section show that the voltage drops associated with the negative powers are less severe than those associated with the positive powers. In FCR operation, the voltage in tap changer position 4 deviates from the nominal voltage by more than $2 \%$ for about 100 seconds only once in the year under consideration.

Thus, despite the significant differences in annual load curves between the auxiliary power transformer and the FCR transformer, significantly more tap changer changes are not necessary. 


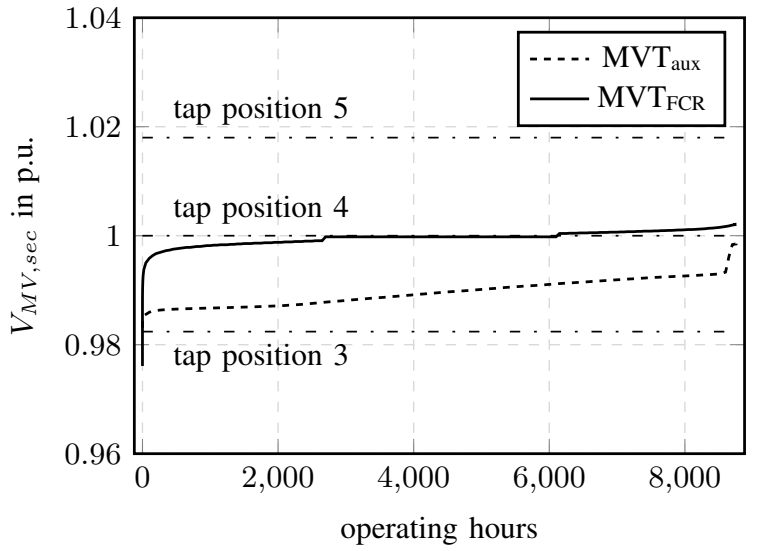

Fig. 7. Ordered annual voltage profiles for auxiliary power transformer (solid line) and FCR transformer (dotted line) for 365 days

If the voltage regulator is configured to be very slow and with a large admissible control deviation, it can compensate for changes in key parameters at the grid connection, which would otherwise cause permanent and distinct voltage changes. With this setup, even large positive frequency deviations would not trigger any switching operations.

With a suitable design, the BESS converters could control the voltage at the grid connection point with the reactive power. Due to the high sensitivity of the voltage with respect to the reactive power, this is possible without significant reduction of the active power.

Further analyses show that no switching is to be expected at the high voltage transformer due to the higher transformation ratio. Overall, no limit cycles between the voltage regulators of the high and medium voltage transformer are to be expected.

\section{CONCLUSION}

In this article, we presented the model-based methodology with which we have shown that during FCR operation on the considered medium voltage regulating transformer there will be no increased switching compared to operation to cover the power plant's auxiliary power demand. For the analysis we assumed the grid connection as a single-phase equivalent circuit diagram. We used the equivalent circuit diagram of lossy transformers for the high voltage and medium voltage transformers. We implemented the grid connection model in
OpenModelica and exported the differential equation system to Maple ${ }^{\mathrm{TM}}$ for further analysis. The analyses were verified in simulations with the OpenModelica implementation as well as with a further implementation in Simulink ${ }^{\mathrm{TM}}$.

In a further Rainflow Conunting analysis the duration and intensity of the frequency deviations and the associated voltage deviations could be characterized in order to review the parameterization of the MVT voltage regulator.

\section{REFERENCES}

[1] The European Commision, "Commission Regulation (EU) 2017/1485 of 2 August 2017 establishing a guideline on electricity transmission system operation," Aug. 2017, legislative Body: ENER, COM. [Online]. Available: http://data.europa.eu/eli/reg/2017/1485/oj/eng

[2] P. Stenzel, J. Linssen, M. Robinius, D. Stolten, V. Gottke, H. Teschner, A. Velten, and F. Schäfer, "Energiespeicher," BWK: das EnergieFachmagazin, vol. 71, pp. 33-48, 2019.

[3] Deutsche Übertragungsnetzbetreiber, "Eckpunkte und Freiheitsgrade bei Erbringung von Primärregelleistung," Apr. 2014. [Online]. Available: https://www.regelleistung.net/ext/download/eckpunktePRL

[4] V. Crastan, Elektrische Energieversorgung 1: Netzelemente, Modellierung, stationäres Verhalten, Bemessung, Schalt- und Schutztechnik, 4th ed. Springer Vieweg, 2015. [Online]. Available: https://www.springer.com/de/book/9783662459843

[5] J. Machowski, J. W. Bialek, and J. R Bumby (James Richard), Power system dynamics: stability and control / Jan Machowski, Janusz W. Bialek, James R. Bumby., 2nd ed. Oxford: John Wiley, 2008.

[6] M. Lindner, R. Witzmann, O. Marggraf, S. Laudahn, B. Engel, S. Patzack, H. Vennegeerts, A. Moser, M. Gödde, F. Potratz, and A. Schnettler, "Ergebnisse der FNN-Studie zu neuen Verfahren der statischen Spannungshaltung," in Zukünftige Stromnetze für erneuerbare Energien : 2. OTTI Konferenz. Regensburg: Ostbayerisches Technologie-Transfer-Institut e.V. (OTTI), Jan. 2015, pp. 104-109, backup Publisher: Zukünftige Stromnetze für Erneuerbare Energien, Berlin (Germany), 27 Jan 2015 - 28 Jan 2015. [Online]. Available: https://publications.rwth-aachen.de/record/465290

[7] "VDE-AR-N 4105 Anwendungsregel: 2018-11 - Generators connected to the low-voltage distribution network," Nov. 2018.

[8] J. Faiz and B. Siahkolah, Electronic Tap-changer for Distribution Transformers, ser. Power Systems. Berlin Heidelberg: SpringerVerlag, 2011. [Online]. Available: https://www.springer.com/de/book/ 9783642199103

[9] J. H. Harlow, Electric Power Transformer Engineering. CRC Press, Aug. 2003.

[10] DIgSILENT, Two-Winding Transformer (3-Phase), ser. PowerFactory 2019 Technical Reference Documentation. 72810 Gomaringen, Germany: DIgSILENT GmbH, Jul. 2019.

[11] D. Oeding and B. R. Oswald, Elektrische Kraftwerke und Netze, 7th ed. Berlin Heidelberg: Springer-Verlag, 2011. [Online]. Available: https://www.springer.com/de/book/9783642192463

[12] “OpenModelica." [Online]. Available: https://openmodelica.org/

[13] "Simscape Electrical ${ }^{\mathrm{TM}}$ " Natick, Massachusetts. [Online]. Available: https://de.mathworks.com/products/simscape-electrical.htm

[14] "Maple 2019," Waterloo, Ontario. [Online]. Available: https://www. maplesoft.com/ 